\title{
Magnetotransport in antidot arrays
}

\author{
D. Weiss *, K. Richter ' , E. Vasiliadou, G. Lütjering \\ Max-Planck-Institut für Festkörperforschung. D-70569 Stuttgart, (iermany \\ (Received 7 May 1993: accepted for publication 28 July 1993)
}

\begin{abstract}
We study magnetotransport in lateral two-dimensional superlattices with periods on the order of $1(0) \mathrm{nm}$. Pronounced low-field transport anomalies reflect the interplay of two characteristic lengths of the system, the cyclotron radius $R_{\mathrm{c}}$ and the period $a$. Measurements at very low temperatures reveal quantum oscillations, periodic in the magnetic field $B$, which are superimposed upon the commensurability anomalies in the magnetoresistance $\rho_{x x}$. We show that the latter effect can be interpreted in terms of quantized periodic orbits which takes into account the chaotic nature of the classical phase space.
\end{abstract}

\section{Introduction}

Todays semiconductor technology provides a means to confine electrons in "boxes" with nanometer dimensions in all three spatial directions. Electron transport through these "artificial atoms" where the nuclear charge is replaced by the confining potential displays unique behavior and is used to probe the "atomic levels" of such a quantum dot structure [1]. In analogy, the coupling of quantum dots into a planar artificial lattice opens up the possibility to study the physical properties of artificial crystals. Just as individual quantum dots are characterized by energy levels analogous to atomic levels, artificial crystals possess a band structure, where the properties of the constituent "atoms" are controlled by the

\footnotetext{
* Corresponding author.

I Present address: Division de Physique Théorique, Institu1 de Physique Nucléaire. 91406 Orsay Cedex, France.
}

superimposed periodic (confining) potential. Two examples of possible periodic potential shapes. both of them experimentally realized, are sketched in Fig. 1. For a weak periodic potential the electron energy (Fermi energy) is well above the potential modulation (Fig. 1a), whereas for a strong potential the Fermi energy intersects the repulsive potential peaks (Fig. 1b). Due to the characteristic shape of the latter type of potential, complementary to the one used to confine electrons in a quantum dot, we call it antidot potential [2-4]. Such artificial crystals allows one to search for qualitatively new phenomena not accessible in natural crystals. One prominent example in this context is the fractal energy spectrum of Bloch electrons in a magnetic field [5-7].

Recently, it has been observed that the magnetoresistance $\rho_{x x}$ displays $1 B$-periodic low field oscillations when a high-mobility $2 D E G$ is subjected to a weak periodic potential $[8-11]$. A weak (sinusoidal) periodic potential transforms the discrete Landau levels into Landau bands 
a

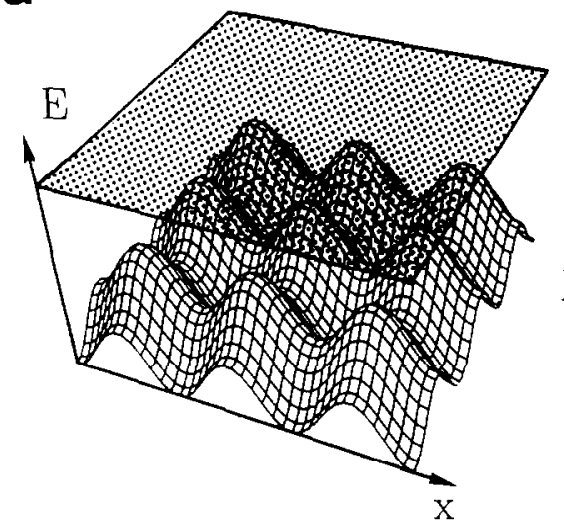

b

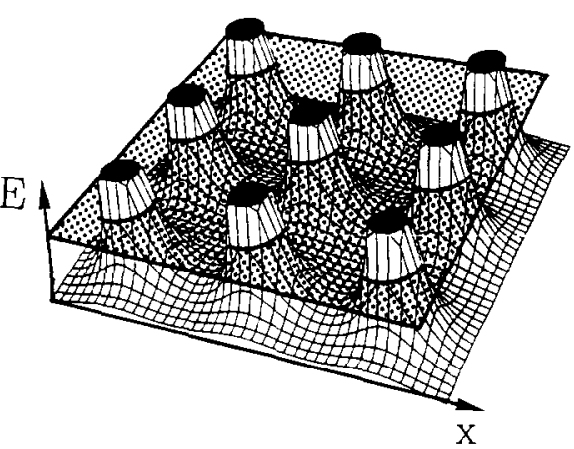

Fig. 1. (a) Weak and (b) strong modulation of the conduction band edge in the $x-y$ plane of the two-dimensional electron gas. The position of the Fermi energy is depicted by the dotted planes.

$[12,13]$. The resulting band structure can be calculated perturbatively for both one- and two-dimensional periodic potentials (see Ref. [7] and references therein). The oscillating bandwidth with minima at $2 R_{\mathrm{c}}=(\lambda-1 / 4) a$ is the origin of the observed oscillatory behavior of $\rho_{x x}$ (and $\rho_{y y}$ ). Here, $R_{\mathrm{c}}$ is the classical cyclotron radius at the Fermi energy $\epsilon_{\mathrm{F}}$, and $\lambda$ is an integer oscillation

a)

b)
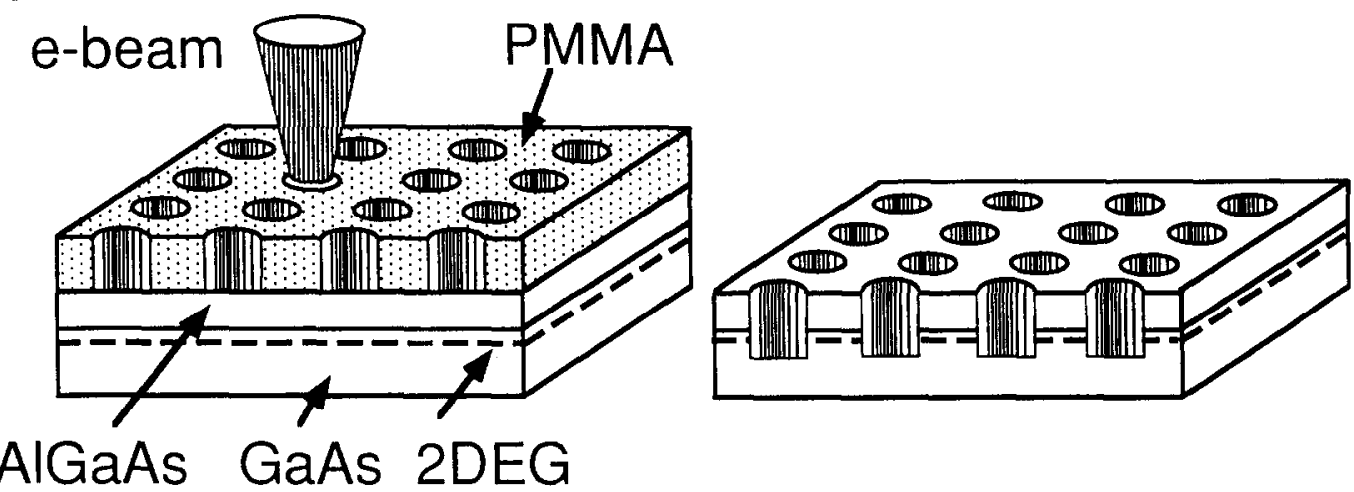

\section{AlGaAs GaAs 2DEG}

c)

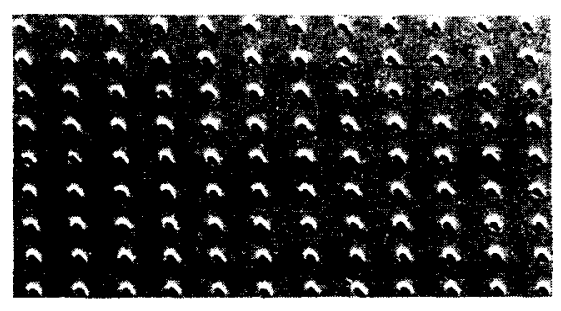

d)

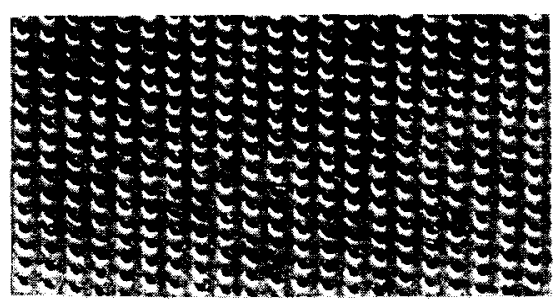

Fig. 2. Fabrication (schematically) of an antidut array by e-beam lithography (a) and subsequent etching of the holes thruugh the $2 D E G$ (b). An electron micrograph of a $300 \mathrm{~nm}$ square antidot lattice tilted by $45^{\circ}$ is shown in (c), a $200 \mathrm{~nm}$ lattice in (d). 
index. The picture of Landau band formation breaks down in an antidot lattice: the strong potential mixes Landau levels with different quantum numbers $n$, and a complicated band structure emerges.

Here we summarize our experimental results on magnetotransport in antidot arrays and sketch our current understanding of the phenomena observed. For a more complete collection of important related work, we refer to recent review articles [14-16]. Before going on to the experimental results, it is useful to specify some characteristic quantities of the system. Distinct transport anomalies stem from the commensurability between the cyclotron radius $R_{\mathrm{c}}$ and the period $a$. In a $2 D E G$ the cyclotron radius, $R_{\mathrm{c}}=$ $\hbar_{1} 2 \pi n_{s} / e B$, is directly connected to the carricr density $n_{s}$ and the magnetic field $B$. In order to observe these effects it is necessary that the electron mean free path, $l_{\mathrm{e}}=l_{\mathrm{F}} \tau$, dependent on the Fermi velocity $l_{F}$ and the Drude relaxation time $\tau$, is much longer than the period $a$. The Fermi wavelength $\lambda_{\mathrm{F}}=\overline{2 \pi / n_{\mathrm{s}}}$, which is a measure of the extent of the wavefunction at zero field, however, is still smaller than the period $a$ which is typically between 200 and $400 \mathrm{~nm}$. Hence, electron transport can be treated in a semi-classical picture where the electrons bounce like balls ballistically through the antidot lattice (for related phenomena see also Ref. [15]). Classically, the motion of a particle in an antidot type of potential is known to be chaotic [17]. We show below that this classical electron dynamics leaves distinct marks in the experiments. In terms of an artificial band structure we face a situation where many bands are occupied; their number increases quadratically with the period $\sim a^{2} n_{\mathrm{s}} / 2$. For antidot periods larger than the Fermi wavelength $\lambda_{\mathrm{F}}$ the electrons in such artificial crystals obey semiclassical rules.

\section{Fabrication of antidot arrays}

The starting point for the fabrication of lateral surface superlattices is a high-mobility two-dimensional electron gas (2DEG) formed at the interface of GaAs/AlGaAs heterojunctions. The high mobility is an essential ingredient which assures that the electron mean free path, $l_{\text {.. }}$ is much longer than the period $a$ of the superimposed periodic potential. Typical mean free paths range from 5 to $10 \mu \mathrm{m}$ before patterning. The nanofabrication process is sketched in Fig. 2. The periodic array of antidots is written into the PMMA-resist layer by using a highly focused electron beam (Fig. 2a). Besides a square arrangement of antidots with periods from $a=200$ $\mathrm{nm}$ to $a=400 \mathrm{~nm}$, we have also fabricated triangular and rectangular arrays. After development, the holes in the PMMA are transferred into the heterojunction by reactive ion etching techniques [18]. After etching through the electron gas we expect an effective potential for the electrons similar to the one sketched in Fig. $1 \mathrm{~b}$; the conduction band is bent up at the antidot positions (due to charged surface states) and defines forbidden regions for the electrons. The effective antidot diameter $d$ consists of the lithographic diameter plus a surrounding depletion region which can be reduced by brief illumination [19]. The antidot array is part of a conventional Hall bar geometry sketched in the top inset of Fig. 3b.

\section{Magnetotransport experiments}

In Fig. 3 we compare the magnetoresistance $\rho_{x x}$ and the Hall resistance $\rho_{x y}$ of patterned and unpatterned areas of the device. Pronounced low-field anomalies dominate the $\rho_{x x}$ and $\rho_{x y}$ trace of the antidot array in a regime where the transport coefficients are usually described by the Drude expressions $\rho_{x x}=m^{*} / e^{2} n_{s} \tau$, and $\rho_{x y}=$ $B / e n_{\mathrm{s}}$ with the electron effective mass $m^{*}$. A double peak structure in $\rho_{x,}$ for $B$ values where $R_{\mathrm{c}} \approx 0.5 a$ and $R_{\mathrm{c}} \approx 1.5 a$ holds documents striking deviations from the simple Drude results. The $\rho_{x x}$ peaks are accompanied by (nonquantized) steps in the Hall resistance displayed in Fig. 3b. At higher $B$ values where the cyclotron diameter becomes smaller than the period $a, \rho_{x \rightarrow}$ drops quickly, Shubnikov-de Haas $(\mathrm{SdH})$ oscillations commence, and $\rho_{x y}$ begins to display quantized Hall plateaus. In this high field regime the traces of patterned and unpatterned segments become 
essentially identical indicating that the intrinsic high mobility is preserved between the antidots. $\Lambda t$ zero field, however, the mobility is limited by scattering off the antidots and the corresponding mean free path becomes comparable to the antidot spacing $a$. A remarkable anomaly in the $\rho_{x y}$-trace is the quenching of the Hall resistance close to $B=0$; the magnified quench is displayed in the inset of Fig. 3b. We address this effect in morc detail below.

The number of peaks and steps resolved in the low $B$ regime critically depends on the effective diameter $d$ and the period $a$ of the antidots. The zero-field resistance is directly controlled by the normalized cross section $d / a$ and the carrier density $n_{\mathrm{s}}$. In Fig. 4 we compare the magnetore-

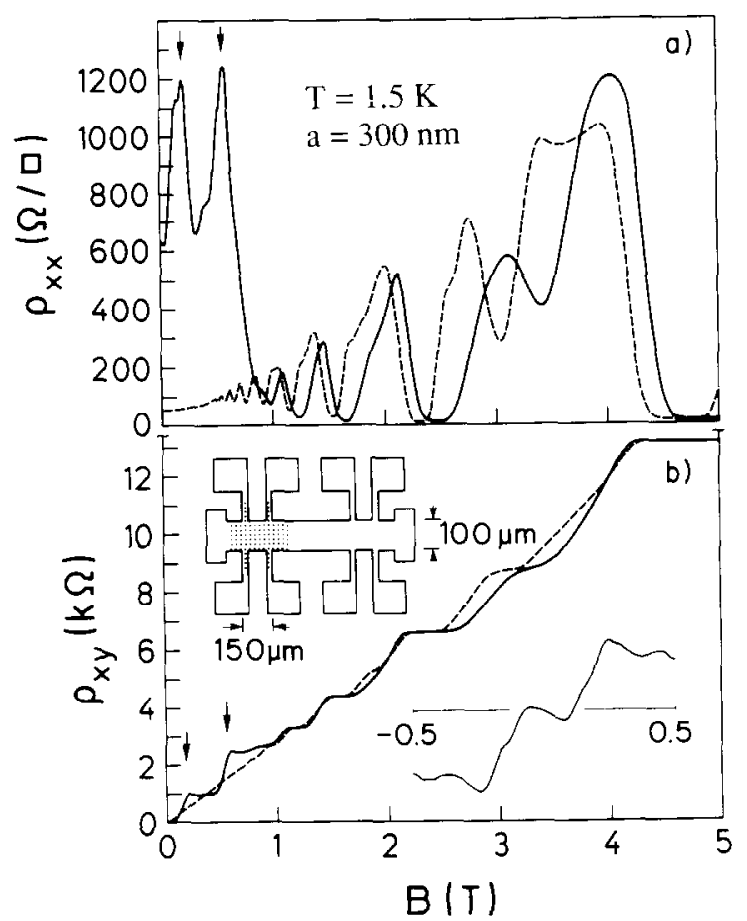

Fig. 3. (a) Magnetoresistance and (b) Hall resistance in patterned (solid line) and unpatterned (dashed line) sample segments at $1.5 \mathrm{~K}$ after brief illumination. In the patterned segment, $n_{\mathrm{s}}$ (determined from the periodicity of the Shubnikov de Haas oscillations at higher $B$ ) is slightly higher after illumination $\left(n_{\mathrm{s}}=2.4 \times 10^{11} \mathrm{~cm}^{-2}\right)$. The arrows mark magnetic field positions where $R_{\mathrm{c}} / a \approx 0.5$ and 1.5 . Top inset of (b): Sketch of the sample geometry. Buttom inset: Magnification of the quench in $\rho_{x y}$ about $B=0$. From Ref. [4].

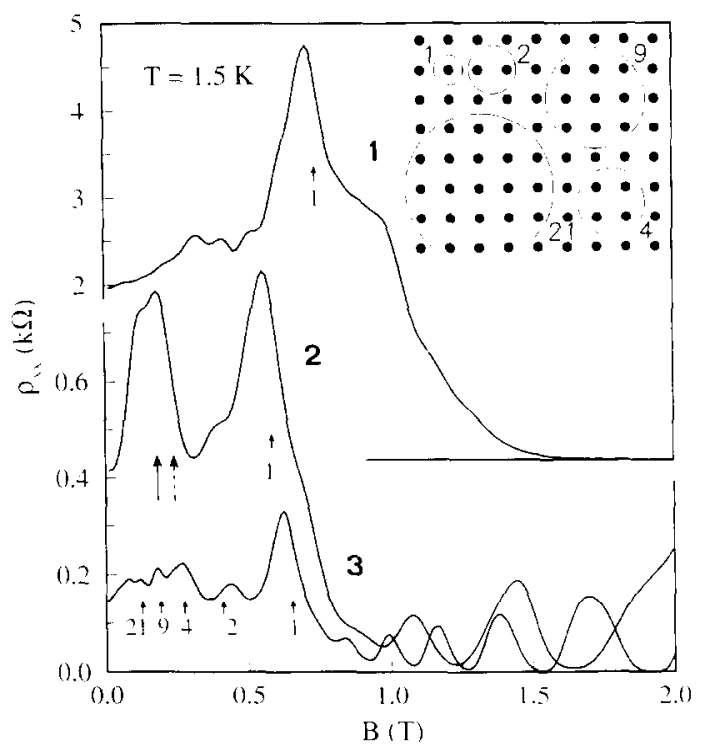

Fig. 4. Low- $B$ anomalies from three different samples. For smaller $d / a$ more structure in $\rho_{x x}$ evolves. All peaks in trace 3 can be ascribed to commensurate orbits around 1, 2, 4, 9 and 21 antidots, as is sketched in the inset. Corresponding $R_{\mathrm{c}} / a$ values, marked by arrows, are $0.5,0.8,1.14,1.7$ and 2.53 , respectively. The dashed arrow for trace 2 marks the position of an unperturbed cyclotron orbil around four antidots $\left(R_{\mathrm{c}} / a=1.14\right)$. The shift of the corresponding resistance peak towards lower $B$ indicates the deformation of the cyclotron orbit in a "soft" potential [17]. From Ref. [4].

sistance curves of three samples with periods $a=200 \mathrm{~nm}$ (top trace) and $a=300 \mathrm{~nm}$ (bottom traces). In curves with smaller zero-field resistance a progressively greater number of peaks becomes resolved. Sample 3 exhibits the largest sequence of new $\rho_{x x}$ peaks and $\rho_{x y}$ plateaus. At each peak, $R_{\mathrm{c}}$ can be associated with a commensurate (circular) orbit encircling a specific number of antidots sketched in the inset of Fig. 4. Although the low-field maxima in trace 3 are well described by unperturbed circular cyclotron orbits, the simple orbit analysis fails to explain the prominent low $B$ peak in curve 2 : the $R_{\mathrm{c}} / a$ value of $\sim 1.5$ deduced from the peak position at $\sim 0.18 \mathrm{~T}$ is not commensurate with the lattice. Model calculations show that this distinct peak stems from electrons on chaotic trajectories trapped on paths around four antidots [17]. Compared to the free electron case our the "orbitaround-four-antidots-peak" is shifted towards 
lower $B$ and provides a first experimental signature of chaotic electron dynamics.

The sequence of peaks observed in experiment reflects the geometry of the antidot lattice. In Fig. 5 we display $\rho_{x x}$ data taken from a rectangular and hexagonal antidot lattice; a characteristic sequence of peaks reflects the particular "crystallographic" arrangement of the antidots. In a rectangular lattice the magnetoresistance strongly depends on the direction of current flow as can be seen from the data in Fig. 5a. The peak corresponding to an orbit around one antidot can only be probed if the current flows along the larger
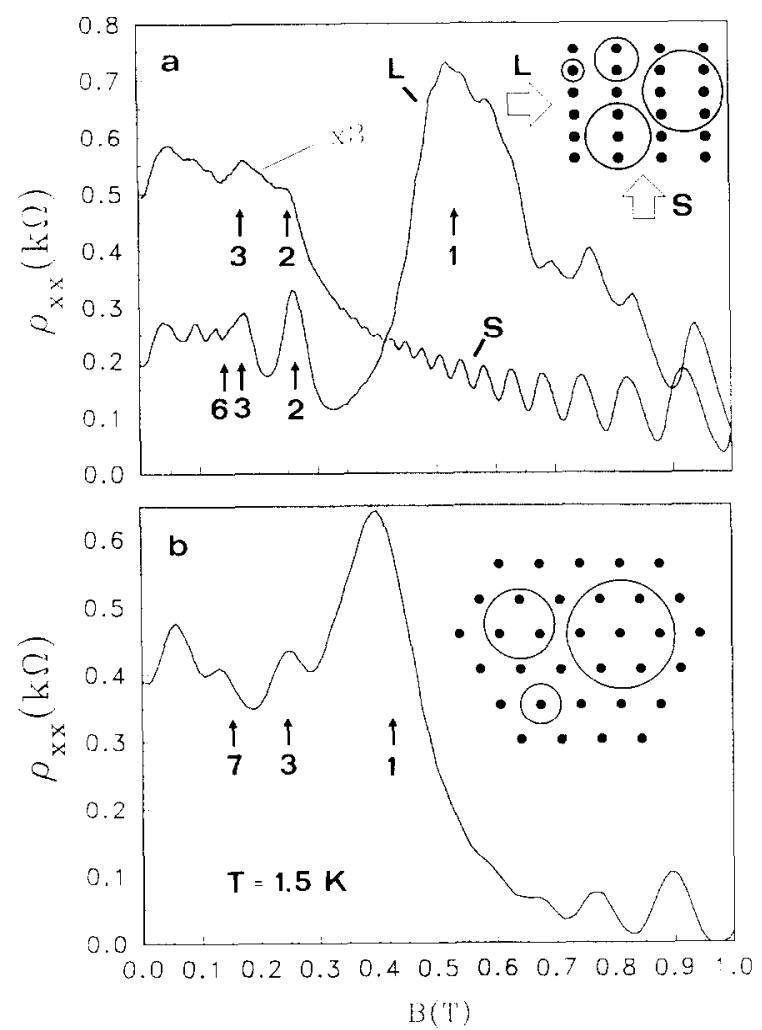

Fig. 5. (a) Magnetoresistance in a rectangular $\left(a_{x}=790 \mathrm{~nm}\right.$, $\left.a_{y}=410 \mathrm{~nm}\right)$ and (b) hexagonal $(a=410 \mathrm{~nm})$ antidot array. In (a) " $L$ " and " $S$ " indicate the direction of current flow with respect to the long and the small period of the lattice as is sketched in the inset. $B$-positions corresponding to free cyclotron orbits around $1,2,3$ and 6 antidots for $R_{\mathrm{c}} / a=0.5,1$, 1.5 and 1.83 , respectively, are marked. In the hexagonal lattice (b) arrows mark orbits around 1,3 and 7 antidots at $R_{\mathrm{c}} / a$ values $0.5,0.87$ and 1.37 .

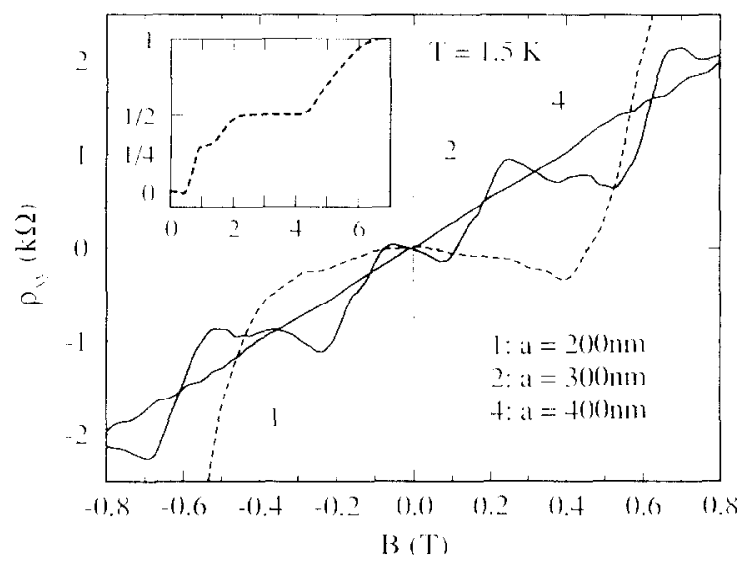

Fig. 6. Qucnching of $\rho_{x y}$ around $B=0$ for three samples with different periods $a$. For the sample with the smallest period $a=200 \mathrm{~nm}$ we find the quench over a $B$-range of nearly $1 \mathrm{~T}$. High field $\rho_{x y}$-data (in units of $h / e^{2}$ ) of sample 1 up to $7 T$ are displayed in the inset. Sample 2 exhibits a negative slope of $\rho_{x y}$ around $B=0$ indicating electron motion opposite to the Lorentz force.

lattice constant. The transport anomalies in such rectangular antidot lattices have been studied by Schuster et al. [20]. The magnetoresistance in a hexagonal lattice, displayed in Fig. 5b, shows peaks when the cyclotron orbit fits around 1,3 , and 7 antidots (see also Refs. [21,22]), hence reflecting the most stable commensurate orbits in this geometrical arrangement.

The quenching of the Ilall resistance in antidot lattices came as a surprise. While such quenching has been observed in small, mesoscopic junctions where the electron motion between the sample boundaries can take place ballistically [23], the antidot array itself is macroscopic; its dimensions are large compared to $l_{e}$. In Fig. 6 we display the data of three antidot samples with different periods (but with comparable antidot diameter): the magnetic field range over which the Hall resistance is quenched decreases with increasing lattice constant. For $a=$ $400 \mathrm{~nm}$ the $\rho_{x y}$ trace around $B=0$ becomes very close to the result expected from the Drude formulas. The quenching of the Hall resistance is just another example which underlines the importance of the classical chaotic electron dynamics discussed in the next section. 


\section{Pinball model and chaotic electron dynamics}

The fact that some of the peak positions in $\rho_{x x}$ can be associated with circular commensurate orbits has stimulated an explanation based on a modified Drude picture, denoted as the pinball model [4]. Such a semi-classical description is supported by the experimental fact that the transport anomalies reported so far continue to be manifested up to high temperatures $T \approx 50 \mathrm{~K}$. The basic assumption of the model is that electrons on commensurate orbits do not contribute to transport since they are trapped for a long time in the vicinity of an antidot. For such impaled orbits the repulsive potential at each antidot provides a local restoring force against drift induced by clectric ficlds. The "removal" of an electron from transport requires a long lifetime of an electron on a pinned orbit, obtained when the mobility between the antidots is preserved. In this picture, the "reduction" of the carrier density for commensurate magnetic fields increases both $\rho_{x x}$ and $\rho_{x y}$. The model involves three diffcrent pools of electrons: pinned, scattered, and drifting ones which are sketched in Fig. 7a. While the fraction $f_{\mathrm{p}}$ of pinned orbits reduces the carrier density for commensurate $B$, the fraction of scattered electrons, $f_{s}$, carries the current at low $B$. These electrons scatter as in a pinball game; their scattering time $\tau^{\prime}$ is of the order $a / v_{\mathrm{F}}$. At higher $B$, both pinned and scattered trajectories die out and become replaced by drifting ones which, due to the suppression of backscattering sketched in Fig. $7 \mathrm{~b}$, are characterized by the intrinsic scattering time $\tau \gg \tau^{\prime}$ of the 2DEG. This is the origin of the pronounced negative magnetoresistance observed in experiment when $R_{\text {c }}$ becomes smaller than $a / 2$. The size of the different fractions depends on the magnetic field and has to be determined numerically. For these calculations we assumed a hard wall potential: hence the electrons between the antidots move on circles or arcs with cyclotron radius $R_{\mathrm{c}}$. Each pool of carriers is characterized by a specific Drude resistivity. In a patterned sample each contingent contributes to the total resistivity which is obtained from the inverted sum of the individual conductivity tensors [4]. The result of a

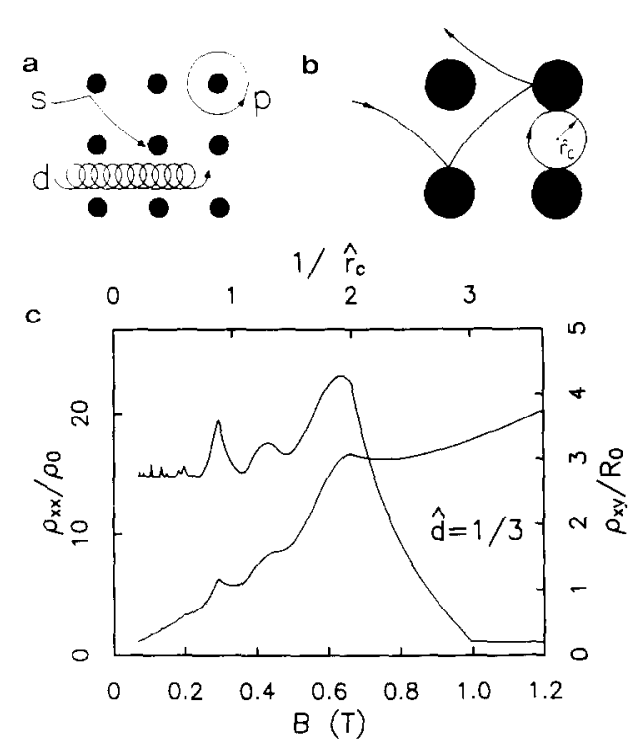

Fig. 7. (a) scattered (s), pinned (p) and drifting (d) trajectories. (b) Trajectories which connect neighboring antidots are suppressed once the cyclotron radius $R_{c}$ becomes smaller than $(a-d) / 2$. The suppression of backscattering is the origin of the negative magnetoresistance observed in experiment. (c) Calculated $\rho_{x x}$ and $\rho_{x y}$ traces for the parameters of sample 3 . Features attributed to orbits around 1,2 and 4 antidots (at $R_{\mathrm{c}} / a=0.5,0.8$ and 1.14 ) are most prominent. The top axis is given in units of the normalized cyclotron radius $\hat{r}_{c}=R_{c} / a$. From Ref. [4].

model calculation with parameters relevant for trace 3 of Fig. $3(d / a=1 / 3)$ is shown in Fig. 7c. Remarkable similarities with the experiment are evident: the commensurability effects associated with orbits around 1, 2, and 4 antidots are quite prominent in both $\rho_{x x}$ and $\rho_{x y}$.

Not explained by the model, however, is the magnetic field position of the low $B$ peak of sample 2 in Fig. 3 and the quenching of the Hall effect. This is due to the omission of an essential ingredient in our model: the finite slope of the potential around each antidot which causes deviations from a circular cyclotron motion. The pinball model works as long as the length over which the antidot potential varies is small compared to $a-d$ : then deviations from strictly circular trajectories occur only in the immediate locale of each antidot. For larger $d / a$ (higher zero field resistance) the validity of the pinball model becomes more and more questionable. In general, the 
electron dynamics in a "soft" antidot potential can not be described in terms of circular cyclotron motion characteristic for conventional electron gases in the limit $\omega_{\mathrm{c}} \tau \gg 1\left(\omega_{\mathrm{c}}=\mathrm{eB} / \mathrm{m}^{*}\right.$ : cyclotron frequency). One way to obtain information about the electron dynamics is to solve the classical equations of motion in a model antidot potential. Using such an approach Lorke et al. [24] calculated the conductivity tensor from the Einstein relation. Fleischmann, Geisel and Ketzmerick (FGK) applied a Kubo-type ansatz [17]. Using a model potential $U(x, y)=U_{0}[\sin (\pi x / a)$ $\sin (\pi y / a)]^{\beta}$ sketched in Fig. 8a they start from the classical Hamiltonian

$$
H=\epsilon_{\mathrm{F}}=\frac{1}{2 m^{*}}(p-e A)^{2}+U(x, y)
$$

( $\boldsymbol{p}$ is the electron momentum and $A$ the vector potential) and integrate the Hamiltonian equations of motion. The numerical results are trajectories $x(t), y(t)$, and corresponding velocities $\iota_{x}(t)=\dot{x}, \iota_{y}(t)=\dot{y}[17]$. The pliase space is generally divided into regions with regular cyclotronlike motion and chaotic motion. This is illustrated

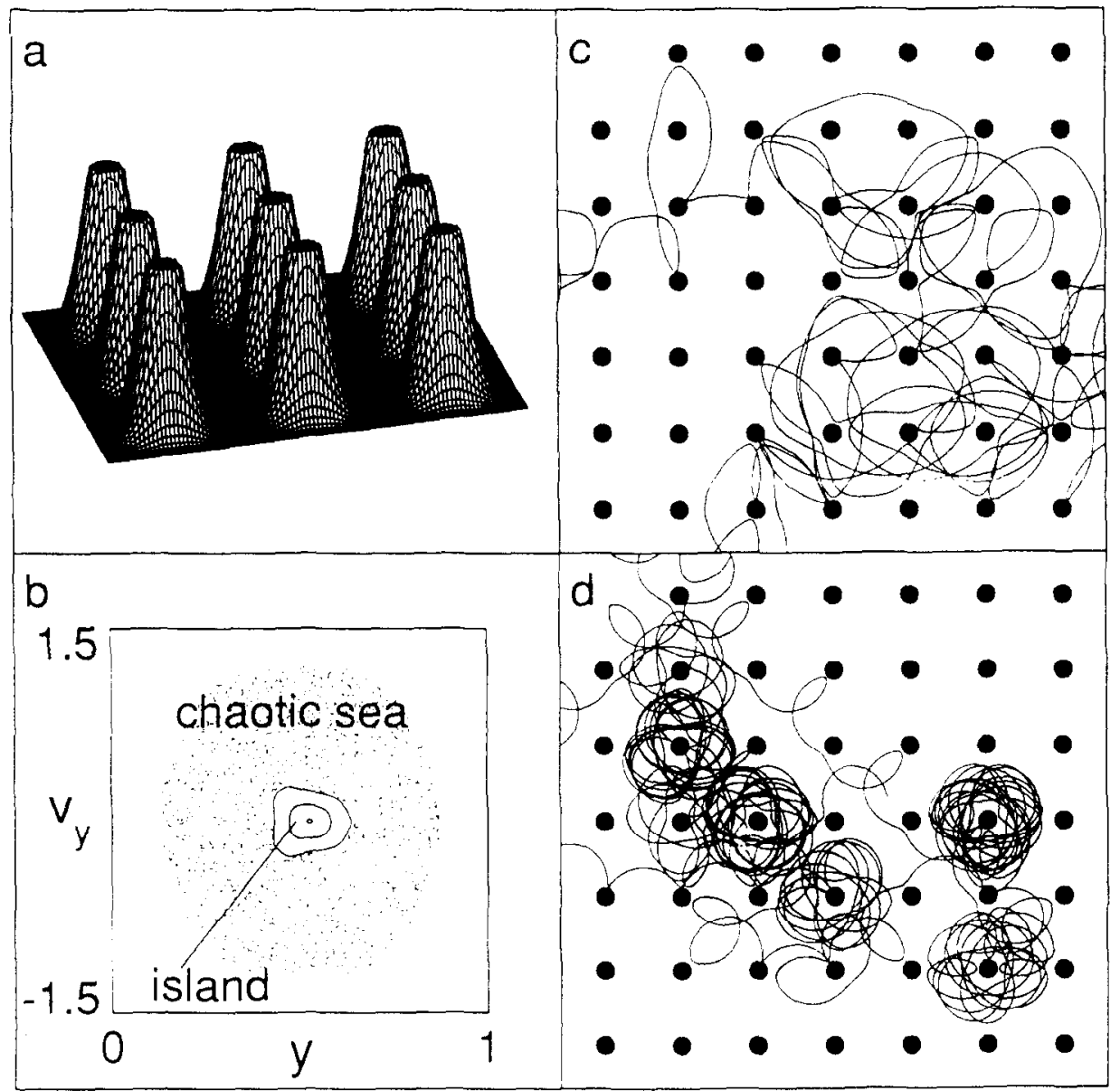

Fig. 8. (a) Antidot model potential. The steepness of the potential is controlled by the parameter $\beta$; here: $\beta=4$. (b) Phase space map showing regions of regular and chaotic motion. The point in the center represents an intersection with with a cyclotron-type orbit of radius $\sim 0.5 a$ around one antidot at $(x, y)=(0,0)$. (c) Real space trajectories for an incommensurate $\left(R_{c}=a\right)$ and $(\mathrm{d})$ commensurate $\left(2 R_{\mathrm{c}}=a\right)$ magnetic field value [28]. 
in the Poincaré surface of section $\left(y, v_{y}\right)$ at $x(\bmod a)=0$ in Fig. $8 \mathrm{~b} ;$ an island of regular motion is clearly distinguishable from the sea of chaotic motion. The points surrounding the stable island stem from one chaotic electron trajectory scattered throughout the antidots lattice. Figs. 8c and 8d display chaotic real space trajectories close to the island of regular motion for an incommensurate and commensurate magnetic field value, respectively. The electrons within the stable island behave as the pinned electrons of our simple pinball model: they do not contribute to transport [17]. Surprisingly, however, Fleischmann et al. found that it is not primarily pinned orbits but chaotic trajectories whirling around the antidots (displayed in Fig. 8d) at commensurate $B$ 's which arc responsible for the emcrgence of the $\rho_{x x}$ peaks. Their contribution to the conductivity $\sigma$ can be expressed via the Kubo-type formula

$\sigma_{i j} \propto \int_{0}^{\infty} \mathrm{e}^{-t / \tau}\left\langle v_{i}(t) v_{j}(0)\right\rangle \mathrm{d} t$.

The indices $i, j$ stand for $x$ or $y$ and the bracket denotes averaging over phase space. The integral is taken only over chaotic trajectories. Note that the unperturbed chaotic motion is "cut off" by the intrinsic scattering time $\tau$. Straightforward evaluation of equations based on Eq. (2) results in $\rho_{x x}$ [17] and $\rho_{x y}$ traces [25] which can be compared with experiment. The classical electron dynamics leaves distinct marks found in experiment. The shift of the "orbit-around-four-antidots-peak" (Fig. 3, trace 2) towards lower magnetic fields can be reproduced within the FKG model, and reflects "squeezed" orbits around four antidots, connected to an augmented cyclotron frequency. The FKG model also successfully describes the quenching of the Hall effect: the suppression of the Hall voltage is due to trajectories channeling over many periods in positive and negative direction along the main axes of the (square) antidot array. This guiding, and therefore the quenching, is less significant if the lattice is more open (larger periods, see Fig. 6). The calculated Hall resistance $\rho_{x y}$ involving the chaotic trajectories via Eq. (2) shows a quenching of the Hall effect and even a negative Hall resis- tivity (due to preferential scattering) as in experiment [25].

\section{Magnetotransport experiments: quantization of periodic orbits}

The magnetotransport experiments described in the previous sections were carried out at temperatures where quantum effects in the low magnetic field range in which the commensurability effects dominate were negligible. In this section, we explore transport in a temperature regime where the quantization of classical electron motion comes into play $[26,27]$. Measurements of $\rho_{x x}$ at $T \approx 0.4 \mathrm{~K}$ display quantum oscillations superimposed upon the low $B$ resistance anomalies. Corresponding data for sample 1 with $d / a \approx 0.5$ are shown in Fig. 9a, where we compare $\rho_{x x}$ from both patterned and unpatterned sample segments. In the unpatterned part, $1 / B$-periodic Shubnikov-de Haas (SdH) oscillations reflect the Landau energy spectrum. The quantum oscillations in the antidot segment reveal quite different behavior. The oscillations are periodic in $B$ with a period $\Delta B \approx 0.105 T \approx h / e a^{2}$ corresponding to the addition of approximately one flux-quantum through the antidot unit cell. At $4.7 \mathrm{~K}$, the quantum oscillations are smeared out while the characteristic $\rho_{x x}$ peak at $2 R_{\mathrm{c}}=a$, attributed to trapped electrons whirling around one antidot (see Fig. 8d), persists. The oscillations periodic in $B$ dominate only the low $B$ regime $\left(2 R_{\mathrm{c}}>a-d\right)$; at high $B$, the sample behaves as if unpatterned, and $\rho_{x x}$ displays minima which are $1 / B$-periodic reflecting quantization of essentially unperturbed cyclotron orbits (Fig. 9a, left inset). In Fig. 9b we plot the oscillation index $\eta$ for both the high and low field regime versus inverse magnetic field positions of the $\rho_{x x}$ minima. At high $B, \eta$ is the filling factor $\nu=n_{\mathrm{s}} h / e B$ counting the number of occupied (spin-split) Landau levels. At low $B$ the antidot potential strongly mixes different Landau levels and the filling factor looses its physical meaning.

Deviations from a linear $1 / B$ dependence in Fig. $9 \mathrm{~b}$ can be assigned to the periodic orbits shown for an intermediate and low $B$ value in the 
insets of Fig. $9 \mathrm{~b}$. These orbits play a central role in explaining the positions of the $\rho_{x x}$ extrema. This is evident from the calculated action $\tilde{S}(B)$ (see below) of these orbits, displayed in Fig. 9b. $B$-periodic oscillations as shown in Fig. 9a, however, are not an inherent property of antidot

a)

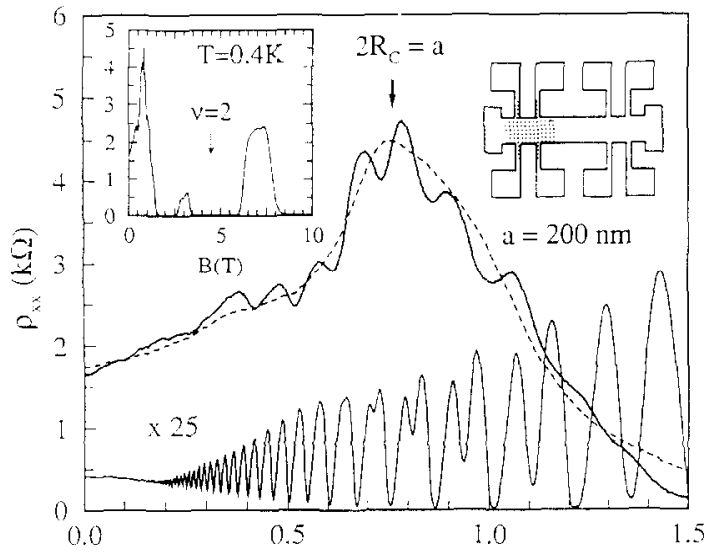

b)

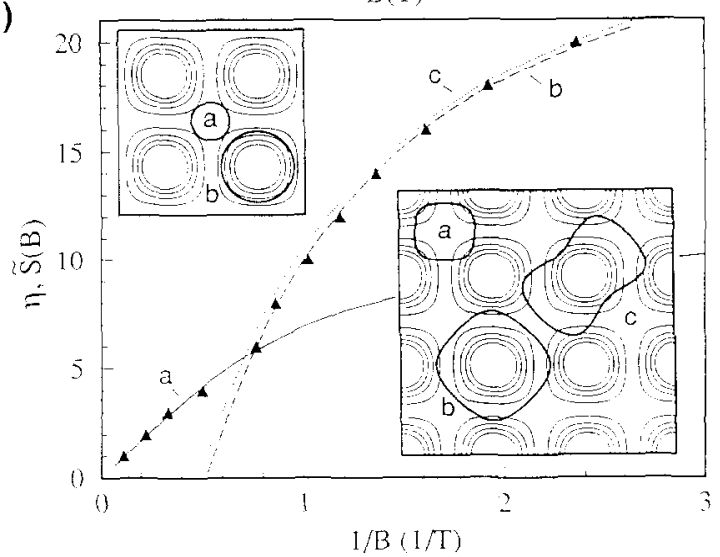

Fig. 9. (a) $\rho_{x x}$ measured in the patterned (top traces) and unpatterned (bottom trace) segment of the same sample for $T=0.4 \mathrm{~K}$ (solid lines) and $4.7 \mathrm{~K}$ (dashed line). The left inset displays the $\rho_{x x}$ trace from the patterned segment up to $10 \mathrm{~T}$; the filling factor $\nu=2$ is marked. At high $B$, the emergence of $\mathrm{SdH}$ oscillations reflects the quantization of essentially unperturbed orbits. Right inset: sketch of the sample layout. (b) The triangles mark all (up to $10 \mathrm{~T}$ ) $1 / B$ positions of the $\rho_{x x}$ minima. At high $B$ the resistance minima lie equidistant on the $1 / B$ scale; at low $B$ the spacing becomes periodic in $B$. Solid, dashed and dotted lines are calculated reduced actions $\tilde{S}(B)$ of orbits (a) (b) and (c), respectively. These orbits are shown for $1 / B 0.6 \mathrm{~T}^{-1}$ (top) and $1 / B=2.7 \mathrm{~T}^{-1}$ (bottom inset). The potential steepness in the calculation was $\beta=2$. From Ref. [26].

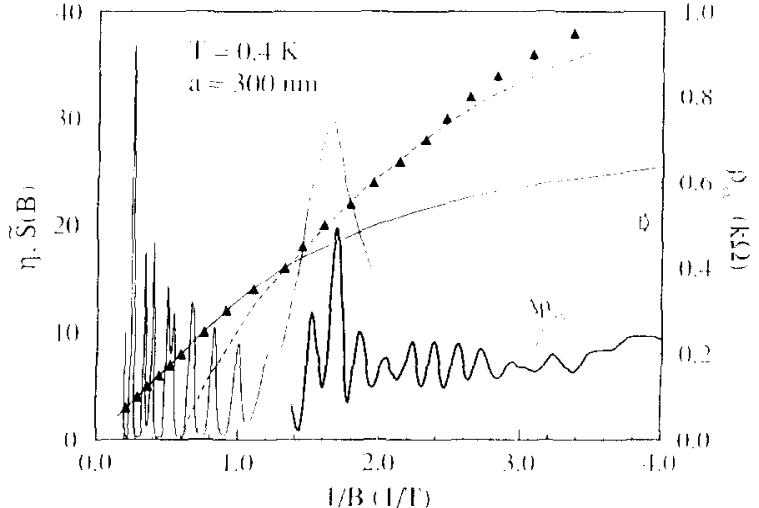

Fig. 10. $\rho_{x x}$ and $\Delta \rho_{x, y}$ (arbitratry units) vs. $1 / B$. The oscillation index $\eta$ labels $\rho_{x x}$ minima positions on the $1 / B$ scale (triangles). Dashed line: Calculated action $\tilde{S}(B)$ for an orbit between four antidots. Solid line: $\tilde{S}(B)$ for electron encom passing one antidot. From Ref. [26].

arrays. Data taken from a superlattice with smaller $d / a \approx 0.4$ are shown in Fig. 10 . In contrast to the data of Fig. 9a the $\rho_{x x}$ minima are periodic in $1 / B$ over nearly the entire field range but display similar deviations for intermediate $B$ where the low field anomalies begin to disappear (between 0.6 and $1 \mathrm{~T}$ ). Again, the experimental data are best described by the action calculated for an orbit between four, and around one, antidot. The crossover from $B$-periodic to $1 / B$-periodic oscillations, dependent on the normalized cross section $d / a$, together with the fact that the antidot array is much larger than the phase breaking length in a 2DEG [29], suggest that the $B$-periodic oscillations observed in experiment are not the usual Aharanov-Bohm oscillations observed, for example, in metal rings. This picture is in contrast to previous work $[14,30]$. In the following we assume that, as usual for quantum transport, $\rho_{x x} \propto d^{2}(E, B)$ probes the electron density of states $d(E, B)$ at the Fermi energy $\epsilon_{\mathrm{r}}$ [31].

To obtain information about $d(E, B)$ one can go through quantum mechanical calculations [32] and obtain complicated spectra which are hard to interpret. Another approach is to use the method of semi-classical quantization of chaotic systems developed by Gutzwiller and others [33]. The problem to be solved is to quantize the electron motion in a classical phase space dominated by 
chaotic trajectories like the ones displayed in Figs. 8c and 8d. It turns out that a smoothed density of states of such a chaotic system can be obtained from its periodic orbits. The orbits which turned out to be relevant in our system (see Refs. [26] and references therein) are displayed in the insets of Fig. 9b. Due to finite temperatures and impurity broadening effects we need to consider only the shortest periodic orbits. Note that these orbits are of negligible measure in a sea of nonperiodic motion. The periodic orbits cause a modulation of $d(E, B)$ with maxima given by the quantized action

$$
\begin{aligned}
S(B) & =\oint\left(m^{*} v+e A\right) \mathrm{d} \boldsymbol{r} \\
& =m^{*} \oint v \mathrm{~d} \boldsymbol{r}-e B \mathscr{A}(B)=2 \pi \hbar N,
\end{aligned}
$$

where $N=(n+\gamma / 2+\alpha / 4)$ contains the quantum number $n$, the winding number $\gamma$ and the Maslov index $\alpha . B \mathscr{A}(B)$ in Eq. (3) is the enclosed flux through a periodic orbit and $v$ is the electron velocity given by Eq. (1). To compare with experiment we calculate the reduced action $\tilde{S}(B)$

$$
\begin{aligned}
& \tilde{S}(B) \equiv 2 \frac{S(B)}{h}-\gamma(B)-\frac{\alpha}{2}-1=2 n ; \\
& n=1,2, \ldots,
\end{aligned}
$$

where $2 n$ now labels minima in $d(E, B)$. Note, that the $y$-axis of Figs. $9 \mathrm{~b}$ and 10 represents $\tilde{S}$, where even $\eta$ are described by Eq. (4). At high $B, 2 n$ is the filling factor $\nu$. The calculated traces of $\tilde{S}(B)$ are shown in Figs. $9 \mathrm{~b}$ and 10 . The three periodic orbits, displayed in the insets of Fig. $9 b$, are sufficient to explain the minima positions of $\rho_{x x}$ These are (i) an orbit between four antidots, denoted as (a), (ii) an orbit around one antidot, (b), and (iii) orbit (c) emerging from a bifurcation of orbit (b).

The $\tilde{S}(B)$ curves in Fig. 9b and 10 differ in the steepness of the model potential; we use $\beta=2$ and $d / a=0.5$ for the solid, dashed and dotted lines in Fig. $9 \mathrm{~b}$, and $\beta=4$ and $d / a=0.4$ for the traces in Fig. 10. Since $d / a$ values are taken from the experiment [4] $\beta$ is the only free parameter.

The magnetic field dependence of $\tilde{S}(B)$ can be explained in a simplified approach evaluating the
$B$ dependence of the enclosed area $\mathscr{A}$. For unperturbed cyclotron motion $S(B)=e B \mathscr{A}$ holds, $\mathscr{A}(B)=\pi R_{\mathrm{c}}^{2}$ scales with $1 / B^{2}$, and $1 / B$-periodic resistance oscillations result. At high $B$, orbit (a) is essentially unperturbed and in this realm oscillations periodic in $1 / B$ are prominent in Figs. $9 b$ and 10 . At lower $B$ where $2 R_{\mathrm{c}}$ is comparable to the period $a$, an essentially unperturbed cyclotron motion requires a sufficiently "open" antidot lattice (small $d / a$, large $\beta$ ). $1 / B$-periodic oscillations between $1 / B=1.3 \mathrm{~T}^{-1}$ and $2.5 \mathrm{~T}^{-1}$ in Fig. 10 document such behavior. Deviations from $\mathscr{A}(B) \propto 1 / B^{2}$ destroy the $1 / B$-periodicity: smaller action is caused by impeding the expansion of a cyclotron orbit. $B$-periodic oscillations result when $\mathscr{A}$ is independent of $B$. This condition is closely fulfilled by orbit (b) calculated for $\beta=2, d / a=0.5$ and shown in the bottom inset of Fig. 9b. This trajectory encloses an area $\sim a^{2}$ causing the $B$-periodic oscillations with $\Delta B \approx$ $h / e a^{2}$ displayed in Fig. 9a.

\section{Acknowledgements}

The present work was done in close collaboration with P. Grambow, A. Menschig, R. Bergmann, H. Schweizer, M.L. Roukes, K. von Klitzing, K. Eberl, K. Ploog, and G. Weimann. We are grateful to R. Fleischmann, T. Geisel, R.R. Gerhardts, and D. Pfannkuche for many helpful discussions. The work was supported by the German Bundesministerium für Forschung und Technology under grant 01BM121/8.

\section{References}

[1] See related work in the current proceedings.

[2] M.L. Roukes and A. Scherer, Bull. Am. Phys. Soc. 34 (1989) 633.

[3] K. Ensslin and P.M. Petroff, Phys. Rev. B 41 (1990) 12307.

[4] D. Weiss, M.L. Roukes, A. Menschig, P. Grambow, K. von Klitzing and G. Weimann, Phys. Rev. Lett. 66 (1991) 2790.

[5] M. Ya. Azbel, Sov. Phys. JETP 19 (1964) 634.

[6] R.D. Hofstadter, Phys. Rev. B 14 (1976) 2239.

[7] D. Pfannkuche and R.R. Gerhardts, Phys. Rev. B 46 (1992) 12606. 
[8] D. Weiss, K. von Klitzing, K. Ploog and G. Weimann, Europhys. Lett. 8 (1989) 179.

[9] D. Weiss, K. von Klitzing, K. Ploog and G. Weimann, Surf. Sci. 229 (1990) 88.

[10] R.R. Gerhardts, D. Weiss and U. Wulf, Phys. Rev. B 43 (1991) 5192

[11] D. Weiss, A. Menschig, K. von Klitzing and G. Weimann, Surf. Sci. 263 (1992) 314.

[12] R.R. Gerhardts, D. Weiss and K. von Klitzing. Phys. Rev. Lett. 62 (1989) 1173.

[13] R.W. Winkler, J.P. Kotthaus and K. Ploog, Phys. Rev. Lett. 62 (1989) 1177.

[14] D.K. Ferry, Prog. Quantum Electron. 16 (1992) 251.

[15] C.W.J. Beenakker and H. van Houten, in: Solid State Physics, Eds. H. Ehrenreich and D. Turnbull (Academic Press, Boston, MA, 1991) p. 1.

[16] W. Hansen, J.P. Kotthaus and U. Merkt, in: Semiconductors and Semimetals, Eds. R.K. Willardson, A.C. Beer and E.P. Weber (Academic Press, San Diego, CA, 1992) p. 279.

[17] R. Fleischmann, T. Geisel and R. Ketzmerick, Phys. Rev. Lett. 68 (1992) 1367.

[18] D. Weiss, P. Grambow, K. von Klitzing, A. Menschig and G. Weimann, Appl. Phys. Lett. 58 (1991) 2960.

[19] G. Müller, D. Weiss, K. von Klitzing, K. Ploog, H. Nickel, W. Schlapp and R. Lösch, Phys. Rev. B 46 (1992) 4346.

[20] R. Schuster, K. Ensslin, J.P. Kotthaus, M. Holland and C. Stanley, Phys. Rev. B 47 (1993) 6843.
[21] H. Fang and P.J. Stiles, Pliys. Rev. B 41 (1990) 10171.

[22] T. Yamashiro, J. Takahara, Y. Takagaki, K. Gamo, S. Namba, S. Takaoka and K. Murase, Solid State Commun. 79 (1991) 885.

[23] M.L. Roukes, A. Scherer, S.J. Allen, Jr., H.G. Craighead, R.M. Ruthen, E.D. Beebe and J.P. Harbison, Phys. Rev. Lett. 59 (1987) 3011.

[24] A. Lorke, J.P. Kotthaus and K. Ploog, Phys. Rev. B 44 (1991) 3447.

[25] R. Ketzmerick, R. Fleischmann and T. Geisel, preprint.

[26] D. Weiss, K. Richter. A. Menschig, R. Bergmann, H. Schweizer, K. von Klitzing and G. Weimann, Phys. Rev. Lett. 70 (1993) 4118.

[27] The influence of an antidot array on $n_{\mathrm{s}}$ extracted from SdH oscillations at high and low $B$ was studied by G.M. Sundaram, N.J. Bassom, R.J. Nicholas, G, J. Rees, P.J. Heard, P.D. Prewett, J.E.F. Frost, G.A.C. Jones, D.C. Peacock and D.A. Ritchie, Phys. Rev. B 47 (1993) 7348.

[28] Figure provided by R. Fleischmann.

[29] A. Yacoby, U. Sivan, C.P. Umbach and J.M. Hong, Phys. Rev. Lett. 66 (1991) 1938.

[30] F. Nihey and K. Nakamura, Physica B 184 (1993) 398.

[31] T. Ando, A.B. Fowler and F. Stern, Rev. Mod. Phys. 54 (1982) 438 .

[32] H. Silberbauer, J. Phys.: Condensed Matter 4 (1992) 7355.

[33] Chaos in Classical and Quantum Mechanics, Ed. M.C. Gutzwiller (Springer, Berlin, 1990). 